Check for updates

Cite this: RSC Adv., 2018, 8, 3657

\title{
Titanate nanotubes-bonded organosulfonic acid as solid acid catalyst for synthesis of butyl levulinate $\uparrow$
}

\author{
Shuolin Zhou, (D) ab Dabo Jiang, ${ }^{a}$ Xianxiang Liu, (D) ${ }^{a}$ Yiping Chen ${ }^{a}$ and Dulin Yin ${ }^{\star a}$ \\ In this study, titanate nanotubes-bonded organosulfonic acid $\left(\mathrm{TNTs}-\mathrm{SO}_{3} \mathrm{H}\right)$ was prepared and employed as \\ an efficient heterogeneous catalyst for esterification of levulinic acid with $n$-butanol. Two reaction products \\ pseudo $n$-butyl levulinate (pseudo-BL) and $n$-butyl levulinate (BL) were detected by GC-MS. The catalyst \\ showed $86.8 \%$ conversion of levulinic acid with $99.7 \%$ selectivity towards $n$-butyl levulinate. TNTs- $\mathrm{SO}_{3} \mathrm{H}$ \\ exhibited strong acidic sites and high stability even after seven cycles of usage and would be well \\ expected to be a potential candidate for alkyl levulinate production.
}

Received 3rd December 2017

Accepted 2nd January 2018

DOI: $10.1039 / \mathrm{c} 7 \mathrm{ra12994g}$

rsc.li/rsc-advances outstanding chemical and physical properties. ${ }^{15-17}$ In addition, TNTs can be easily obtained by hydrothermal treatment of $\mathrm{TiO}_{2}$ particles (TNPs) in $\mathrm{NaOH}$ aqueous solution, followed by a facile post-treatment. ${ }^{18}$ To the best of our knowledge, till date, there are very few reports of titanate nanotubes as an acid-based catalyst support. ${ }^{19}$ Although sulfate- $\mathrm{TiO}_{2}$ nanotubes used as catalysts have exhibited high activity for the esterification of acetic acid, ${ }^{20}$ the challenge of stability occurs in the presence of water due to the detachment of active $\mathrm{SO}_{4}{ }^{2-}$ ions, which leads to activity loss during recycling of the catalysts. Titanate nanotubes, on the other hand, possess large surface area, abundant surface hydroxyl groups and stable tubular structures, which advantageously make them a versatile candidate in the catalysis field. It was found that the potential to modify $\mathrm{TiO}_{2}$ nanotubes to incorporate organosulfonic acid groups will open new opportunities for their use as acid-based catalysts in a variety of reactions.

In this paper, titanate nanotubes-bonded organosulfonic acid was prepared by chemical grafting-oxidation method using (3-mercaptopropyl)trimethoxysilane as precursor and $\mathrm{TiO}_{2}$ nanotubes as support (Scheme 1) and then used as catalyst for the esterification reaction of levulinic acid with $n$-butyl alcohol. The effects of different reaction parameters on the catalytic activity of the catalyst and the reusability of the catalyst after several reaction cycles were evaluated. The as-prepared TNTs$\mathrm{SO}_{3} \mathrm{H}$ catalyst was expected to be a good candidate for alkyl levulinate production from biomass-derived platform molecules.

\section{Experimental}

\subsection{Catalyst preparation}

$\mathrm{TiO}_{2}$ nanotubes were synthesized using a hydrothermal synthesis approach described in literature. ${ }^{18}$ To prepare TNTs$\mathrm{SO}_{3} \mathrm{H}, 4 \mathrm{~mL}$ of (3-mercaptopropyl)trimethoxysilane was added drop-wise to a three-necked round-bottom flask containing 

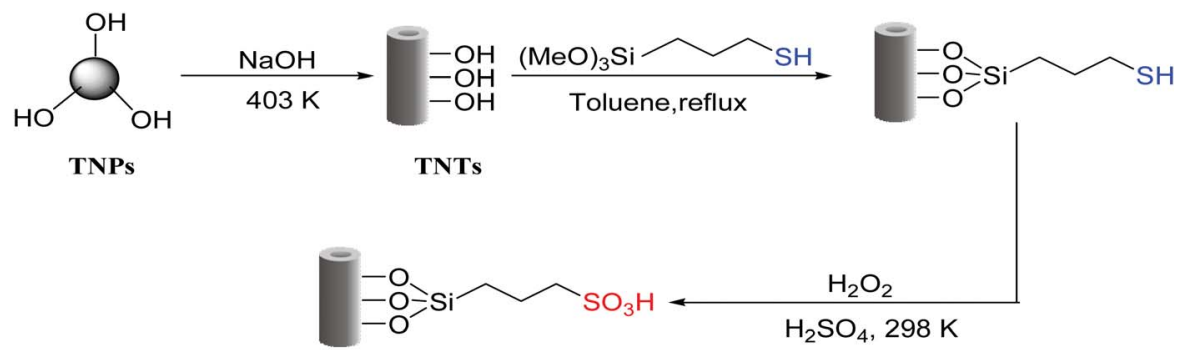

TNTs-SO $\mathrm{O}_{3} \mathrm{H}$

Scheme 1 Synthesis strategy for the preparation of $\mathrm{TNTs}-\mathrm{SO}_{3} \mathrm{H}$ catalyst.

$1.0 \mathrm{~g}$ of TNTs and $15 \mathrm{~mL}$ of dry toluene, and the reaction mixture was refluxed for $24 \mathrm{~h}$ under continuous mechanical stirring. Then, the resultant product was filtered and washed with acetone. Next, the obtained powder was oxidized with $10 \mathrm{wt} \% \mathrm{H}_{2} \mathrm{O}_{2}$ in methanol $(10 \mathrm{~mL})$ at $298 \mathrm{~K}$ for $24 \mathrm{~h}$, followed by treatment with $1 \mathrm{~mol} \mathrm{~L}^{-1} \mathrm{H}_{2} \mathrm{SO}_{4}$ for complete protonation. Finally, the mixture was filtered and washed with $\mathrm{H}_{2} \mathrm{O}$ and acetone to obtain the final TNTs- $\mathrm{SO}_{3} \mathrm{H}$ catalyst.

\subsection{Characterization}

The samples were dispersed in ethanol and poured onto grids for observation. The morphologies of the samples were investigated using transmission electron microscopy (TEM) with an acceleration voltage of $200 \mathrm{kV}$ (JEOL 2100, Japan). $\mathrm{N}_{2}$ adsorption-desorption isotherms were recorded with an ASAP 2400 physisorption instrument made by Micromeritics Corporation (UNITED STATES). Prior to measurement, all samples were dried under vacuum at $333 \mathrm{~K}$ for $24 \mathrm{~h}$. Pore size distribution was calculated by the $\mathrm{BJH}$ method. Fourier transform infrared spectra (FT-IR) of the samples were collected by the $\mathrm{KBr}$ pellet technique on a Nicolet 370 infrared spectrophotometer in the range $500-4000 \mathrm{~cm}^{-1}$. Thermo gravimetric analysis (TGA) curves were recorded in air flow on a Netzsch Model STA 409PC instrument with a heating rate of $20 \mathrm{~K} \mathrm{~min}^{-1}$ from room temperature to $973 \mathrm{~K}$ using $\alpha-\mathrm{Al}_{2} \mathrm{O}_{3}$ as the standard material. The acidity strength of catalyst was determined by Hammett acidity function method combined with UV-visible spectroscopy (Shanghai Tianmei Co. Ltd, China). 4-Nitroaniline was chosen as the basic indicator and $\mathrm{CCl}_{4}$ was chosen as the solvent.

\subsection{Catalytic testing}

In a typical run, the experimental procedure was as follows: $1 \mathrm{mmol}$ of LA and $5 \mathrm{mmol}$ of $n$-butanol together with catalyst ( $5 \mathrm{wt} \%$ of LA) were added to a round bottom flask and heated in an oil bath. At periodic intervals, $0.2 \mathrm{~mL}$ of the reaction solution was withdrawn, centrifuged, and analyzed using an Agilent $6890 \mathrm{~N}$ gas chromatograph (GC). The gas chromatograph was equipped with a HP-5 capillary column $(30 \mathrm{~m}$ length, $0.32 \mathrm{~mm}$ internal diameter, $0.25 \mu \mathrm{m}$ film thickness) and a flame ionization detector (FID). The injection port temperature was $523 \mathrm{~K}$, the oven temperature was $453 \mathrm{~K}$, and the detector temperature was $523 \mathrm{~K}$. Degradation intermediates were identified by GC-
MS, a Shimadzu GC system equipped with a capillary $(30 \mathrm{~m}$ length, $0.32 \mathrm{~mm}$ internal diameter, $0.25 \mu \mathrm{m}$ film thickness). Mass spectrometer conditions were the following: ionization mode: EI, electron energy $70 \mathrm{eV}$, interface temperature: $523 \mathrm{~K}$, ion source temperature: $473 \mathrm{~K}$, mass scan range: $40-640 \mathrm{~m} / \mathrm{z}$, solvent delay $3.0 \mathrm{~min}$. The flow rate of the carrier gas (helium) was $1.0 \mathrm{~mL} \mathrm{~min}{ }^{-1}$. A split ratio of $1: 50$ was used for the injection of $0.2 \mu \mathrm{L}$ of the solutions. The NIST05S.LIB library was used for the mass spectrum analysis.

\section{Results and discussion}

\subsection{Characterization of catalyst}

The morphologies of the TNTs and TNTs- $\mathrm{SO}_{3} \mathrm{H}$ were investigated by employing TEM. As shown in Fig. 1(a), the TNTs reveal a needlelike shape and can be up to several microns in length; each tube tends to have a diameter of approximately $10 \mathrm{~nm}$. Fig. 1(b) shows a typical TEM image of the TNTs- $\mathrm{SO}_{3} \mathrm{H}$. It is
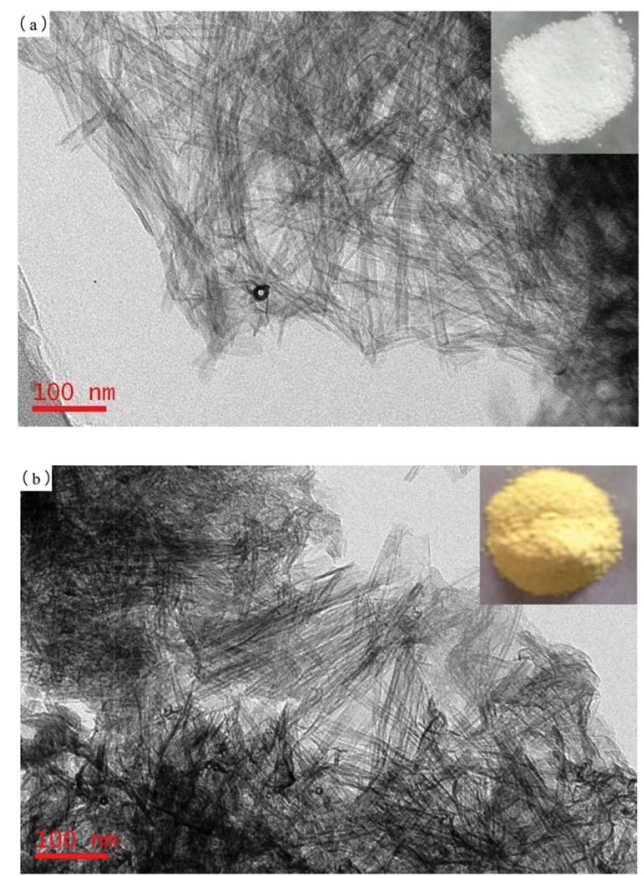

Fig. 1 TEM images of (a) TNTs and (b) TNTs $-\mathrm{SO}_{3} \mathrm{H}$; the insets are the corresponding photographs. 


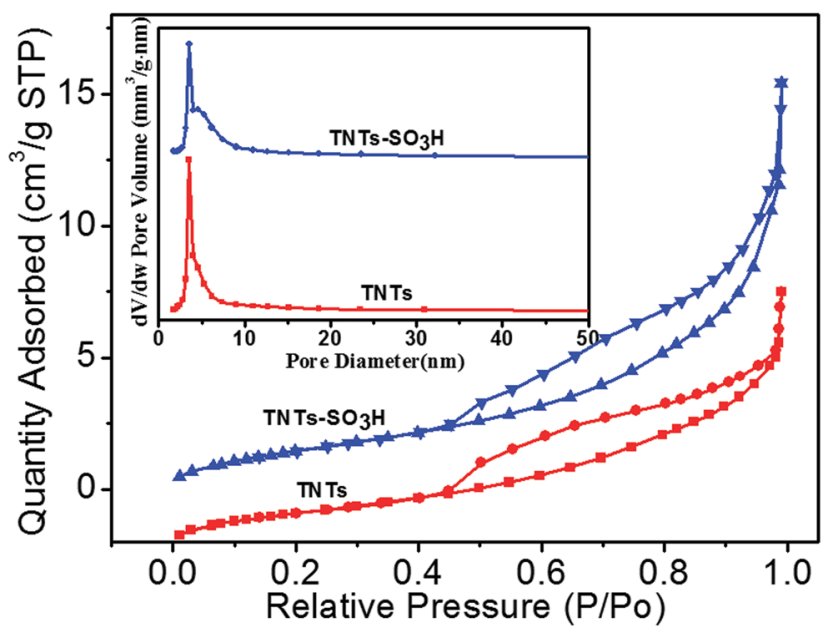

Fig. 2 Nitrogen gas adsorption-desorption isotherms and pore size distribution curves of TNTs and TNTs $-\mathrm{SO}_{3} \mathrm{H}$.

observed that the tubular structure of TNTs does not change after modification.

Fig. 2 presents the nitrogen adsorption and desorption isotherms and the corresponding Barrett-Joyner-Halenda (BJH) pore size distribution curves of the TNTs and TNTs- $\mathrm{SO}_{3} \mathrm{H}$. As shown, it can be observed that the nitrogen adsorption isotherms do not change after modification. The as-prepared TNTs- $\mathrm{SO}_{3} \mathrm{H}$ have a high specific surface area and pore volume of $217 \pm 8 \mathrm{~m}^{2} \mathrm{~g}^{-1}$ and $0.56 \mathrm{~cm}^{3} \mathrm{~g}^{-1}$, respectively. In addition, the obtained pore size distribution plot of $\mathrm{TNTs}-\mathrm{SO}_{3} \mathrm{H}$ shows a narrow microporous distribution with an average pore diameter of $4.8 \mathrm{~nm}$, which are in good accordance with TEM results.

Fig. 3 shows the FT-IR spectra of the pure TNTs and TNTs$\mathrm{SO}_{3} \mathrm{H}$ samples. The FT-IR characterization of TNTs is consistent with those reported in the literature. ${ }^{21}$ The peaks at $3376.9 \mathrm{~cm}^{-1}$ and $1627.7 \mathrm{~cm}^{-1}$ correspond to the $-\mathrm{OH}$ stretching and the bending vibrations of the adsorbed water respectively. In the case of TNTs- $\mathrm{SO}_{3} \mathrm{H}, \mathrm{C}-\mathrm{H}$ stretching vibrations of silylating agent was observed at $2925 \mathrm{~cm}^{-1}$ and $2825 \mathrm{~cm}^{-1}$. Furthermore, the bands at $1040.3 \mathrm{~cm}^{-1}$ and $899.6 \mathrm{~cm}^{-1}$ can be collectively

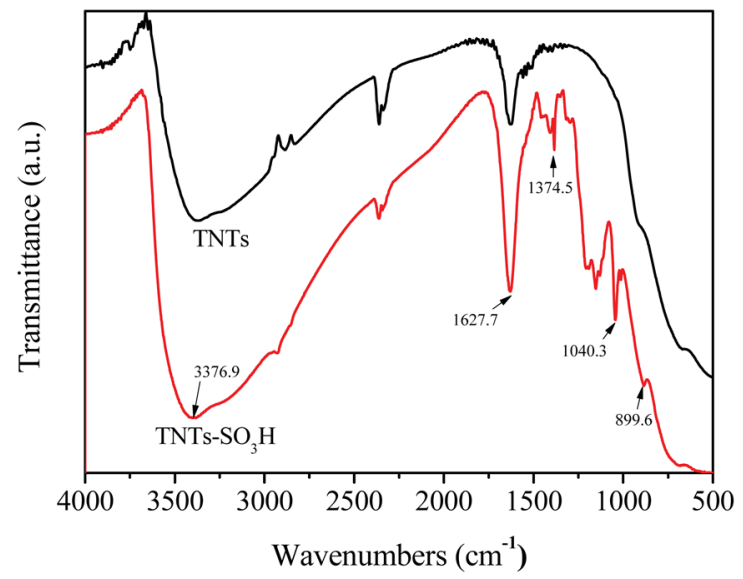

Fig. 3 FT-IR spectra of TNTs and TNTs $-\mathrm{SO}_{3} \mathrm{H}$. attributed to $\mathrm{Si}-\mathrm{O}$ stretching vibrations. The band at $1374.5 \mathrm{~cm}^{-1}$ corresponds to the stretching frequency of $\mathrm{S}=\mathrm{O}$ in $\mathrm{SO}_{3} \mathrm{H}$, which is consistent with the reported IR spectra for $-\mathrm{SO}_{3} \mathrm{H}^{22}$

The thermal stability of TNTs and TNTs- $\mathrm{SO}_{3} \mathrm{H}$ was confirmed by TGA as displayed in Fig. 4. The TGA curve of TNTs shows an initial weight loss (4 wt\%) below $373 \mathrm{~K}$, which corresponds to the loss of the physically adsorbed water. At approximately $425 \mathrm{~K}$, the TG curve was slightly inflected, possibly corresponding to the dehydration of interlayer water. ${ }^{23}$ In the TGA curve of TNTs- $\mathrm{SO}_{3} \mathrm{H}$ a mass loss of approximately $8 \%$ weight occurred between 473 and $823 \mathrm{~K}$, corresponding to the loss of the covalently bound organic group. Simultaneously, from the TGA, it is understood that $\mathrm{TNTs}-\mathrm{SO}_{3} \mathrm{H}$ has greater thermal stability, confirming that it can be used in organic reactions in the range of $353-473 \mathrm{~K}$.

The acidity of the catalyst can be effectively evaluated by determining the Hammett acidity functions $\left(H_{0}\right)$ using UVvisible spectroscopy. The $H_{0}$ can be expressed as:

$$
H_{0}=\mathrm{p} K(\mathrm{I})_{\mathrm{aq}}+\log \left([\mathrm{I}]_{\mathrm{s}} /\left[\mathrm{IH}^{+}\right]_{\mathrm{s}}\right)
$$

where 'I' represents the indicator base (4-nitroaniline), and $[\mathrm{I}]_{\mathrm{s}}$ and $\left[\mathrm{IH}^{+}\right]_{\mathrm{s}}$ are the molar concentrations of the unprotonated and protonated forms of the indicator, respectively. The $\mathrm{p} K(\mathrm{I})_{\mathrm{aq}}$ value of 4-nitroaniline is 0.99 .

The UV-vis spectra of 4-nitroaniline with the concentration of $1.40 \times 10^{-4} \mathrm{~mol} \mathrm{~L}^{-1}$ in $\mathrm{CCl}_{4}$ solution is displayed in Fig. 5. The maximal peak was observed at $329 \mathrm{~nm}$ and the corresponding absorbance value was 1.725. After addition of TNTs- $\mathrm{SO}_{3} \mathrm{H}$, the absorbance value of the unprotonated form of the indicator was decreased to 0.718 , indicating that the indicator is partially in the form of $\left[\mathrm{IH}^{+}\right]_{\mathrm{s}}$. According to the Lambert-Beer's law, the value of $[\mathrm{I}]_{\mathrm{S}} /\left[\mathrm{IH}^{+}\right]_{\mathrm{S}}$ can be determined and calculated. TNTs-SO $\mathrm{S}_{3} \mathrm{H}$ showed $H_{0}$ value of 0.84 . The total acid density of the catalyst determined by $\mathrm{NaOH}$ titration was around $0.50 \mathrm{mmol} \mathrm{g}^{-1}$.

\subsection{Catalytic activity}

In the present study, the reaction products, $n$-butyl levulinate (BL) and pseudo $n$-butyl levulinate (pseudo-BL), were detected

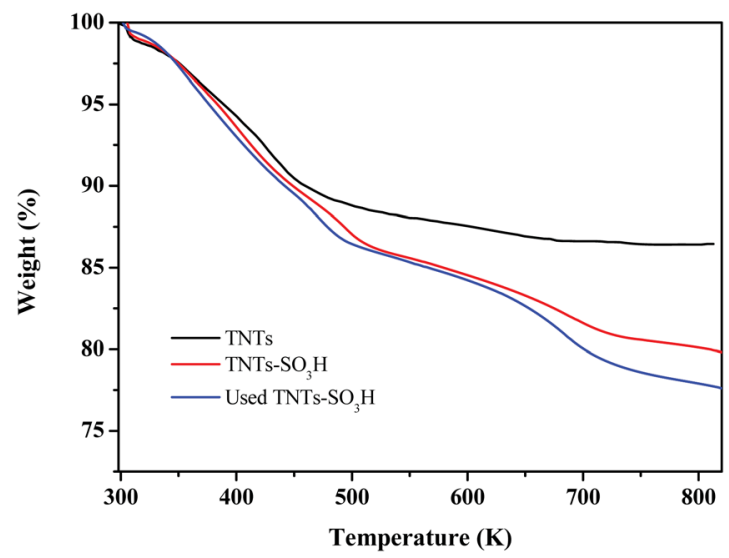

Fig. 4 TGA profile of TNTs and TNTs $-\mathrm{SO}_{3} \mathrm{H}$. 


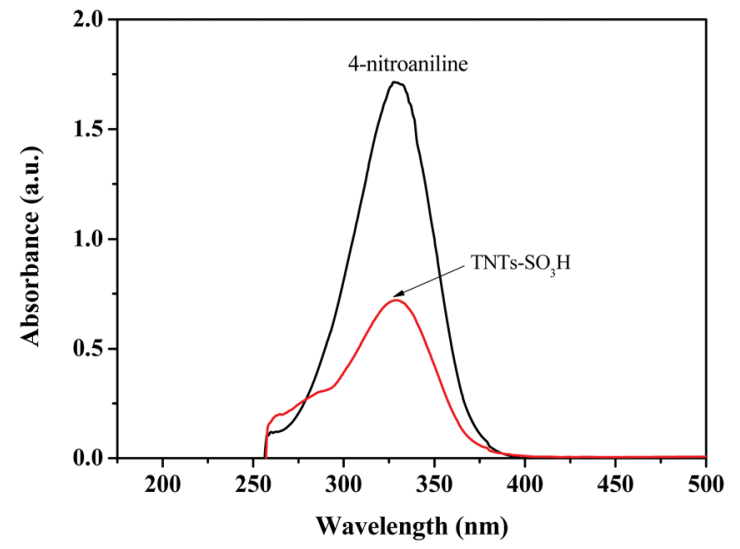

Fig. 5 Absorption spectra of 4 -nitroaniline and $\mathrm{TNTs}-\mathrm{SO}_{3} \mathrm{H}$ in $\mathrm{CCl}_{4}$ solution.

by GC-MS; the corresponding GC-MS spectra are shown in Fig. S1. $\uparrow$ Similar results have recently been found by Ciptonugroho et $a l .{ }^{24}$ The catalytic performances of all the materials, i.e., TNPs, TNTs and TNTs- $\mathrm{SO}_{3} \mathrm{H}$, were investigated for LA conversion and the results are shown in Table 1. Blank experiment was performed without addition of any catalyst, in which the conversation of LA was calculated to be $6.3 \%$ (Table 1, entry 1 ). In contrast, the presence of catalysts resulted in higher product yields than that corresponding to the blank reaction reference, revealing the necessity of an acid catalyst to carry out this reaction. For TNPs, $11.0 \%$ LA conversion with $44.6 \%$ BL selectivity is obtained (Table 1, entry 2). This may be because TNPs as a mild Lewis acid catalysts catalyze this reaction. ${ }^{25}$ The conversion of LA is $15.4 \%$ with $64.9 \%$ BL selectivity when catalyzed by TNTs (Table 1 , entry 3 ). The enhanced reaction activity may be ascribed to the TNTs with open-tubular structure, providing more active sites. However, when TNTs are used as catalyst and the reaction time is further increased to $6 \mathrm{~h}$, the conversion of LA only reaches $25.5 \%$ (Table 1 , entry 4 ). Moreover, TNPs- $\mathrm{SO}_{3} \mathrm{H}$ obtained from TNPs using the same preparation method was used as a catalyst, resulting in a quantitative LA conversion (Table 1, entry 5). This implies that an acid catalyst with a moderate density and the acid strength of the acidic sites might be required to allow this reaction to proceed

Table 1 Catalytic activity over different catalyst samples ${ }^{a}$

\begin{tabular}{|c|c|c|c|c|c|}
\hline \multirow[b]{2}{*}{ Entry } & \multirow[b]{2}{*}{ Catalyst } & \multirow[b]{2}{*}{ Time/h } & \multirow[b]{2}{*}{ LA Conversion/\% } & \multicolumn{2}{|c|}{ Selectivity/\% } \\
\hline & & & & $\mathrm{BL}$ & Pseudo-BL \\
\hline 1 & - & 2 & 6.3 & 36.8 & 63.2 \\
\hline 2 & TNPs & 2 & 11.0 & 44.6 & 55.4 \\
\hline 3 & TNTs & 2 & 15.4 & 64.9 & 35.1 \\
\hline 4 & TNTs & 6 & 25.5 & 80.4 & 19.6 \\
\hline 5 & TNPs-SO ${ }_{3} \mathrm{H}$ & 2 & 27.9 & 81.5 & 18.5 \\
\hline 6 & TNTs-SO ${ }_{3} \mathrm{H}$ & 2 & 37.5 & 84.4 & 15.6 \\
\hline 7 & TNTs-SO ${ }_{3} \mathrm{H}$ & 4 & 48.2 & 97.0 & 3.0 \\
\hline
\end{tabular}

${ }^{a}$ Experimental conditions: $1 \mathrm{mmol}$ of LA, $5 \mathrm{mmol}$ of $n$-butanol, $5 \mathrm{wt} \%$ catalyst loading, $383 \mathrm{~K}$. effectively. As for the TNTs- $\mathrm{SO}_{3} \mathrm{H}$, it exhibits higher catalytic activity (Table 1, entries 6 and 7).

It is worth noting that the conversion of LA improves to $48.2 \%$ after $4 \mathrm{~h}$ with $97 \%$ selectivity of $n$-BL. This is due to the grafted accessibility of the $\mathrm{SO}_{3} \mathrm{H}$ sites in catalyst, which is able to facilitate the reaction. Moreover, the dispersing property of catalyst in reaction media was improved. In view of the relative dimensions of $\mathrm{LA}$ and pseudo-BL, the pore openings of TNTs- $\mathrm{SO}_{3} \mathrm{H}$ should allow these molecules to freely diffuse inside the pore system of the TNTs- $\mathrm{SO}_{3} \mathrm{H}$ and reach both internal and external active sites. Overall, the trend in conversion of LA over the prepared catalyst samples is as follows: TNTs- $\mathrm{SO}_{3} \mathrm{H}>$ TNTs $>$ TNPs.

\subsection{Effects of reaction conditions}

The influence of reaction temperature and time on LA esterification over TNTs- $\mathrm{SO}_{3} \mathrm{H}$ was further investigated and the results are shown in Fig. 6. At a lower temperature $(373 \mathrm{~K})$, the conversion of LA is $38.6 \%$ after $4 \mathrm{~h}$ of reaction. The conversion increased to $48.2 \%$ and $59.3 \%$ on carrying out the reaction at 383 and $393 \mathrm{~K}$ respectively. The obtained results show that at high reaction temperature, the reaction had a high conversion. The selectivity of BL increases on increasing the reaction time from 0.5 to $4 \mathrm{~h}$, whereas the selectivity of pseudo-BL initially increases and then decreases with reaction time. This reveals that the pseudo-BL is an intermediate product during the reaction process. At 383 and $393 \mathrm{~K}$, the selectivity of $\mathrm{BL}$ improves to $97 \%$ and $99.3 \%$, respectively. This is probably due to the fact that the efficient collisions between the reactions provide sufficient energy for pseudo-BL conversion into BL at high temperature, and BL selectivity is therefore improved.

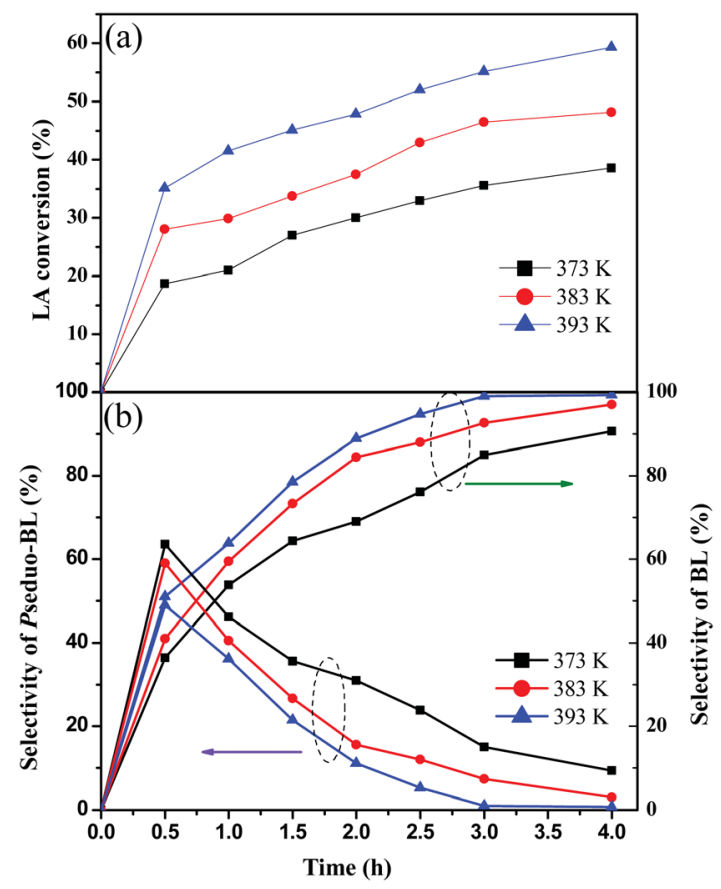

Fig. 6 Change of LA conversion (a) and BL selectivity (b) as function of time over TNTs- $\mathrm{SO}_{3} \mathrm{H}$ catalyst at different reaction temperatures. Experimental conditions: $1 \mathrm{mmol}$ of LA, $5 \mathrm{mmol}$ of $n$-butanol, $5 \mathrm{wt} \%$ catalyst loading. 
We also investigated the effect of catalyst amount at $393 \mathrm{~K}$. LA conversion and BL selectivity rise to $86.8 \%$ and $99.7 \%$ by applying $10 \mathrm{wt} \%$ of $\mathrm{TNTs}-\mathrm{SO}_{3} \mathrm{H}$. The increase in both LA conversion and BL selectivity with an increasing catalyst dosage would be attributed to an increase in the availability and number of catalytically active sites.

\subsection{Reusability of catalyst}

The catalytic stability of the TNTs- $\mathrm{SO}_{3} \mathrm{H}$ was evaluated in the reaction by performing consecutive batch reactions at $393 \mathrm{~K}$ with alcohol acid molar ratio 5:1 and $10 \mathrm{wt} \%$ of catalyst loading. After each catalytic cycle, the catalyst was recovered by filtration, washed with acetone and dried overnight at $333 \mathrm{~K}$ before reuse. As shown in Fig. 7, it is evident that the catalytic activity of $\mathrm{TNTs}-\mathrm{SO}_{3} \mathrm{H}$ was practically the same even after 7 consecutive cycles with around $80 \%$ conversion of LA. In addition, the selectivity of BL on recycled catalyst was almost equal to that over the fresh catalyst. TGA analysis of seven-times used and fresh catalyst are very similar (Fig. 2), evidencing that the $-\mathrm{SO}_{3} \mathrm{H}$ groups were essentially preserved. In addition, TEM image and FT-IR spectra (Fig. S2 and S3 in the ESI $\dagger$ ) of the spent catalyst after repeated experiments show that the TNTs- $\mathrm{SO}_{3} \mathrm{H}$ structure and surface groups have no noticeable change. Even though a slight activity decay was observed, which might be due to mass loss of the catalyst during several recycling runs, LA conversion decreases only from $86.8 \%$ to $81.2 \%$ in 7 cycles. It can be concluded that the catalyst can be reused without considerable loss in activity.

Furthermore, in order to investigate the heterogeneity of the catalytic reaction, a hot filtration experiment was run. After $2 \mathrm{~h}$, the catalyst was separated by centrifugation and the filtrate was further stirred for another $12 \mathrm{~h}$ at $393 \mathrm{~K}$. It was found that no product was detected, indicating that the catalytic reaction is predominantly heterogeneous.

\subsection{Proposal for a possible mechanism}

According to above-obtained results, the mechanism proposed for the conversion of LA to BL is shown in Scheme 2. It is similar

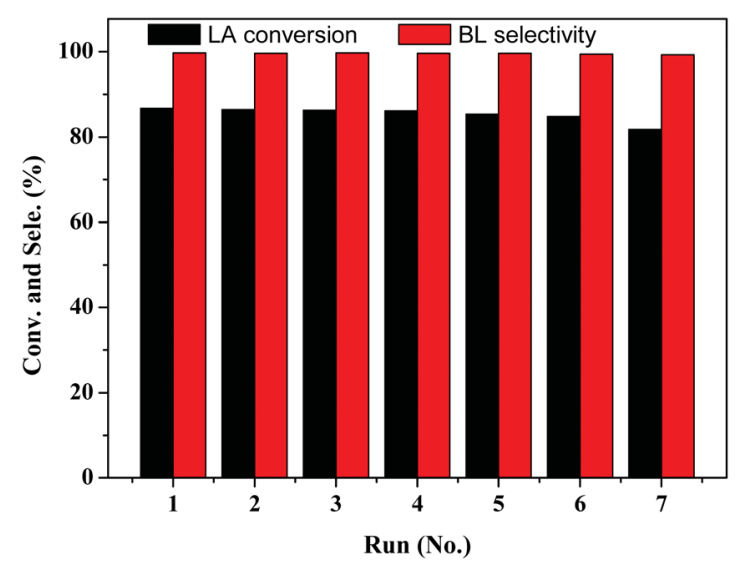

Fig. 7 Stability of the TNTs $-\mathrm{SO}_{3} \mathrm{H}$ on the esterification of LA with $n$ butanol.

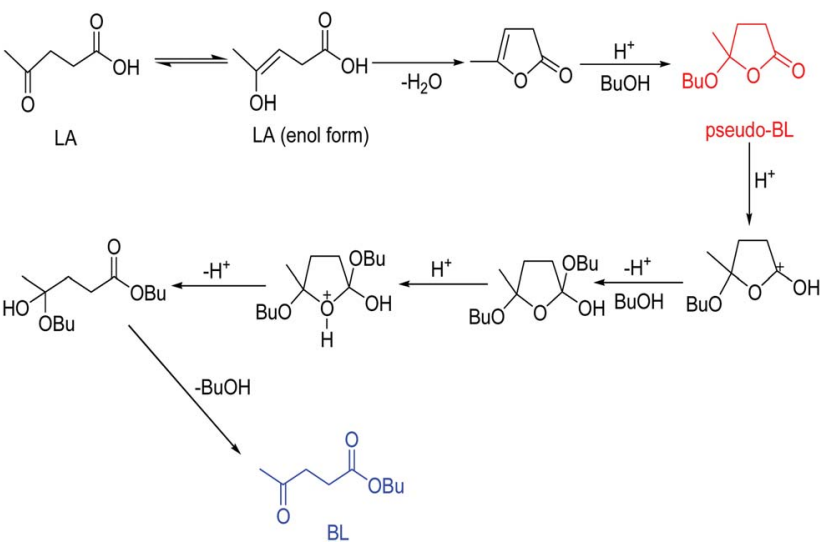

Scheme 2 Proposed possible mechanism for levulinic acid esterification with $n$-butyl alcohol.

to that recently reported by $\mathrm{Al}$-shaal et $a .^{26}$ and Ciptonugroho et $a .^{24}$ The enol form of LA can undergo intramolecular lactonization to afford $\alpha$-angelica lactone, followed by the electrophilic addition of $n$-butanol to obtain the intermediate product pseudo-BL. Subsequently, the protonation of pseudo-BL, in turn, facilitates another nucleophilic addition and ring opening occurs resulting in BL.

\section{Conclusion}

Herein, titanate nanotubes-bonded organosulfonic acid as a novel solid acid catalyst was successfully prepared, and it exhibited good catalytic performance for esterification of biomass-derived LA with $n$-butanol. A high conversion of LA and desired product (BL) selectivity were achieved under relatively mild conditions. It was noted that the catalyst retains its good catalytic performance even after seven consecutive reaction cycles. These results indicated that $\mathrm{TNTs}-\mathrm{SO}_{3} \mathrm{H}$ nanocatalysts are useful as eco-friendly and ideal materials for esterification and biomass-based production.

\section{Conflicts of interest}

There are no conflicts to declare.

\section{Acknowledgements}

This work was supported by the National Natural Science Foundation of China (Grant No. 21606082, 21776068) and Scientific Research Fund of Hunan Provincial Education Department (Grant No. 15C0816, 16C0114).

\section{Notes and references}

1 M. J. Climent, A. Corma and S. Iborra, Green Chem., 2014, 16, 516-547.

2 F. D. Pileidis and M.-M. Titirici, ChemSusChem, 2016, 9, 562582. 
3 K. Yan, C. Jarvis, J. Gu and Y. Yan, Renewable Sustainable Energy Rev., 2015, 51, 986-997.

4 A. Démolis, N. Essayem and F. Rataboul, ACS Sustainable Chem. Eng., 2014, 2, 1338-1352.

5 Y.-H. Chung, T.-H. Peng, H.-Y. Lee, C.-L. Chen and I. L. Chien, Ind. Eng. Chem. Res., 2015, 54, 3341-3354.

6 P. Demma Carà, R. Ciriminna, N. R. Shiju, G. Rothenberg and M. Pagliaro, ChemSusChem, 2014, 7, 835-840.

7 H. J. Bart, J. Reidetschlager, K. Schatka and A. Lehmann, Ind. Eng. Chem. Res., 1994, 33, 21-25.

8 J. Li, X. Wang, G. Chen, D. Li, Y. Zhou, X. Yang and J. Wang, Appl. Catal., B, 2015, 176-177, 718-730.

9 S. Dharne and V. V. Bokade, J. Nat. Gas Chem., 2011, 20, 1824.

10 X. Zhou, Z. X. Li, C. Zhang, X. P. Gao, Y. Z. Dai and G. Y. Wang, J. Mol. Catal. A: Chem., 2016, 417, 71-75.

11 K. Y. Nandiwale and V. V. Bokade, Chem. Eng. Technol., 2015, 38, 246-252.

12 J. Li, D. Li, J. Xie, Y. Liu, Z. Guo, Q. Wang, Y. Lyu, Y. Zhou and J. Wang, J. Catal., 2016, 339, 123-134.

13 E. L. S. Ngee, Y. Gao, X. Chen, T. M. Lee, Z. Hu, D. Zhao and N. Yan, Ind. Eng. Chem. Res., 2014, 53, 14225-14233.

14 Z. Hu, Y. Peng, Y. Gao, Y. Qian, S. Ying, D. Yuan, S. Horike, N. Ogiwara, R. Babarao, Y. Wang, N. Yan and D. Zhao, Chem. Mater., 2016, 28, 2659-2667.
15 D. V. Bavykin, J. M. Friedrich and F. C. Walsh, Adv. Mater., 2006, 18, 2807-2824.

16 P. Roy, S. Berger and P. Schmuki, Angew. Chem., Int. Ed., 2011, 50, 2904-2939.

17 J. Tripathy, K. Lee and P. Schmuki, Angew. Chem., Int. Ed., 2014, 126, 12813-12816.

18 T. Kasuga, M. Hiramatsu, A. Hoson, T. Sekino and K. Niihara, Adv. Mater., 1999, 11, 1307-1311.

19 M. Kitano, K. Nakajima, J. N. Kondo, S. Hayashi and M. Hara, J. Am. Chem. Soc., 2010, 132, 6622-6623.

20 C.-H. Lin, S.-H. Chien, J.-H. Chao, C.-Y. Sheu, Y.-C. Cheng, Y.-J. Huang and C.-H. Tsai, Catal. Lett., 2002, 80, 153-159.

21 T. Kubo and A. Nakahira, J. Phys. Chem. C, 2008, 112, 16581662.

22 S. V. Atghia and S. Sarvi Beigbaghlou, J. Organomet. Chem., 2013, 745, 42-49.

23 Y. Suzuki and S. Yoshikawa, J. Mater. Res., 2004, 19, 982-985. 24 W. Ciptonugroho, M. G. Al-Shaal, J. B. Mensah and R. Palkovits, J. Catal., 2016, 340, 17-29.

25 K. Nakajima, R. Noma, M. Kitano and M. Hara, J. Phys. Chem. $C, 2013,117,16028-16033$.

26 M. G. Al-Shaal, W. Ciptonugroho, F. J. Holzhauser, J. B. Mensah, P. J. C. Hausoul and R. Palkovits, Catal. Sci. Technol., 2015, 5, 5168-5173. 